\title{
SPECTRA OF FAST J-SHOCKS IN DENSE, STAR-FORMING REGIONS
}

C.F. McKee(UC Berkeley), D.J. Hollenbach (NASA Ames), and T. Jernigan (NASA Johnson)

Stellar winds, bipolar outflows, and supernovae generate strong shocks in molecular clouds. If the molecular gas is magnetized and weakly ionized, shocks slower than about 40$50 \mathrm{kms}^{-1}$ (C-shocks) emit primarily in the infrared. Faster shocks, or shocks in a weakly magnetized or more highly ionized medium (J-shocks), emit primarily in the ultraviolet and are thus harder to detect in dusty regions. J-shocks in molecular gas are usually dissociative, so that molecular emission lines from such shocks are produced by molecules formed behind the shock front. Observations of fast shocks in molecular clouds can provide valuable insights into energetic events occurring within them.

We have calculated the structure and spectrum below $10^{4} \mathrm{~K}$ for steady J-shocks with perpendicular magnetic fields $B=10^{-6} n_{0}^{\frac{1}{2}} \mathrm{G}$, where $n_{0}$ is the pre-shock density of hydrogen nuclei. The dominant chemical reactions among $\mathrm{H}, \mathrm{C}, \mathrm{O}$ and the relevant atomic and molecular ions were followed; the ionization balance of $\mathrm{He}, \mathrm{N}, \mathrm{Ne}, \mathrm{Si}, \mathrm{S}, \mathrm{Cl}, \mathrm{Fe}$, and $\mathrm{Ni}$ was also calculated. Atomic and molecular line cooling, allowing for line trapping and dust, competed against $\mathrm{H}_{2}$ formation heating, photoionization, photoelectric heating by grains, and ambipolar diffusion. The basic assumption in this analysis is that after formation on a grain, the $\mathrm{H}_{2}$ molecule is ejected with $4.2 \mathrm{eV}$ of internal energy, with the fraction of this energy delivered to the gas through collisional de-excitation depending on density.

We conclude that:

1. J-shocks in molecular gas are dissociative. If grains survive, $\mathrm{H}_{2}$ re-forms within a column density $\sim 10^{21.5} \mathrm{~cm}^{-2}$.

2. The shock chemistry is determined by the temperature at which $\mathrm{H}_{2}$ re-forms. This temperature is larger at higher density because a larger fraction of the heat of formation of $\mathrm{H}_{2}$ is deposited in the gas. For $T \gtrsim 400 \mathrm{~K}$, chemical activation energies are overcome; $\mathrm{C}$ goes into $\mathrm{CO}$ and the remaining $\mathrm{O}$ goes into $\mathrm{H}_{2} \mathrm{O}$.

3. The strongest IR emission lines from J-shocks are usually atomic fine structure lines such as [OI] $\lambda 63 \mu \mathrm{m}$; by contrast, for $\mathrm{C}$-shocks molecular lines are generally strongest. Heretofore undetected lines such as [Ni II] $\lambda 6.6 \mu \mathrm{m}$ can be quite strong.

4. Detectable radio recombination lines are produced by J-shocks for $n_{0} \gtrsim 10^{5}$ $\mathrm{cm}^{-3}$ and $v_{s} \gtrsim 50 \mathrm{kms}^{-1}$. In principle, these lines allow the discovery of J-shocks in highly obscured regions and permit mapping at high angular resolution.

5. High velocity water masers are naturally produced by $\mathrm{J}$-shocks in dense molecular gas $\left(n_{o} \gtrsim 10^{7} \mathrm{~cm}^{-3}\right)$. The shock chemistry produces a high concentration of $\mathrm{H}_{2} \mathrm{O}\left(\left[\mathrm{H}_{2} \mathrm{O}\right]\right.$ $\sim 3 \times 10^{-4}$ ) at $T \gtrsim 500 \mathrm{~K}$. Infrared line pumping is most likely responsible for producing the maser. 